\title{
Comparison of common tests performance for Mycobacterium bovis infection diagnosis in low prevalence dairy cattle herds of southern Chile
}

\author{
Eduardo Raffo $^{\mathrm{a}, \mathrm{b}}$, Gustavo Montia, Miguel Salgado ${ }^{\mathrm{a}}$
}

\begin{abstract}
The aim of the present study was to assess the performance of routinely used diagnostic tests to detect $M$. bovis infection in cattle populations with very low infection prevalence. A cross-sectional study was performed using 609 animals from 17 herds that were sampled for blood interferon, and tested with the caudal fold tuberculin test (CFT). It was planned that animals which showed CFT positive results were sent to the slaughterhouse for post mortem confirmation testing. However, not all the animals considered in the initial sampling were culled. Paired population proportions of positive results for the different diagnostic tools were compared. Apparent sensitivity and specificity was also estimated. Overall, intradermal tuberculin based diagnostics showed better performance in comparison to interferon based test, even though interferon was slightly more specific. From these results, it may be suggested that specific cut-off lines for interferon based techniques for certain areas with particular low bTB prevalence should be considered, as well as the modification of more specific antigens and the use of better combinations of PPDs if in vitro testing is intended must be taken into consideration. The uncertain role of interference bacteria that could be affecting the results of the in vitro analysis must be considered, and perhaps this could explain the apparent lack of consensus of the results obtained in Chile versus the ones obtained elsewhere. More studies must be performed in order to assess this issue.

Key words: bovine, tuberculosis, prevalence, diagnose.
\end{abstract}

\section{INTRODUCTION}

Bovine tuberculosis (bTB) is a contagious chronic disease with a worldwide distribution, caused by Mycobacterium bovis. It affects most domestic mammals and wild species, although not all species are equally susceptible to this infection (Neill et al 2005). In addition, the infection of cattle with this bacterium has been a concern for public and animal health authorities around the world for a long time (Schiller et al 2011). It corresponds to one of the most important diseases for dairy cattle farmers, responsible for generating clear negative effects on animal production systems. Complete eradication of bTB is not only difficult to achieve in high prevalence countries, but also in countries with low proportions of bTB infected herds, and even in some official tuberculosis free countries, bTB is currently slightly rising (Bezos et al 2014a).

The Chilean bTB eradication program divides the country into two different epidemiological areas, the 'Control Zone' in the North, containing 28\% of the national cattle population, and the 'Eradication Zone' in the South containing $72 \%$ of the country's cattle (Max et al 2011). The most used diagnostic procedure in Chile for the bTB program -as in many countries throughout the world- is the single caudal fold test. This test allows the detection of infected animals and has contributed to a reduction in the prevalence of the infection in a great majority of

\footnotetext{
Accepted: 08.11.2017.

anstituto de Medicina Preventiva Veterinaria, Facultad de Ciencias Veterinarias, Universidad Austral de Chile, Valdivia, Chile.

${ }^{b}$ Escuela de Graduados, Facultad de Ciencias Veterinarias, Universidad Austral de Chile, Valdivia, Chile.

*Corresponding author: M Salgado; miguelsalgado@uach.cl.
}

herds following the traditional "test and slaughter" control strategy. However, the sensitivity and specificity of this test is highly variable (de la Rua-Domenech et al 2006), for example in herds with a low infection prevalence, the Positive Predictive Value has been shown to be very low and the test results are not suitably trustworthy to ensure the eradication of the disease (Schiller et al 2011).

Due to the diagnostic limitations of tuberculin-based tests, other diagnostic procedures were developed to aid the detection of $M$. bovis infection within herds, such as Gamma interferon and ELISA tests. However, the results of these tests might also be influenced by the stage and severity of the disease, individual host factors and prevalence of cross-reacting organisms.

The current situation of the cattle $M$. bovis infection rate in southern Chile is rather low and the estimated herd and within-herd prevalence in this region are 0.3 and $0.67 \%$, respectively (Max et al 2011). Under this epidemiological scenario, we aim to assess the performance of routinely used diagnostic tests to detect $M$. bovis infection in cattle populations with very low infection prevalence.

\section{MATERIAL AND METHODS}

\section{STUDY DESIGN AND ANIMAL POPULATION}

A cross-sectional study using cattle herds located in the Los Ríos Region, Chile, was performed between January 2012 and September 2014. A total of 609 animals from 17 herds were sampled for blood, and tested with the caudal fold tuberculin test (CFT). It was planned that all animals included in this survey were sent to the slaughterhouse for post mortem testing. However, not all the animals considered on the initial sampling were culled. Of the initial population considered on this study, 37.1\% 
of the animals had a complete set of samples (in-vivo and post-mortem). The numbers of animals per herd ranged from 1 to 91, belonging mainly to the Holstein-Friesian breed, with the exception of one Hereford herd. The study design considered animals that belonged to herds with previously known bovine tuberculosis status, which in turn, were divided into: animals with confirmation tests on tissue (post mortem) and those that were diagnosed using field tests (in vivo).

\section{SAMPLE COLLECTION FOR $M$. BOVIS INFECTION DIAGNOSIS}

At least $14 \mathrm{~mL}$ of heparinized blood were obtained by venipuncture from the coccygeal or jugular vein using a vacutainer and transported to the laboratory at room temperature, to be processed within 12 hours using the Gamma Interferon Test (Bovigam®). In addition, in those individuals that were sent to slaughter, retro-pharyngeal or mediastinal lymph nodes were collected for confirmation of $M$. bovis infection using PCR. The samples were kept frozen at $-80^{\circ} \mathrm{C}$ until being processed in the Infectious Diseases Lab at Instituto de Medicina Preventiva Veterinaria, Facultad de Ciencias Veterinarias, Universidad Austral de Chile.

\section{MYCOBACTERIUM BOVIS INFECTION DIAGNOSIS}

Tuberculin test. In all the animals considered for the study, CFT was performed as a screening test to detect animals infected by M. bovis (de la Rua-Domenech et al 2006). Therefore, prior to obtaining a blood sample, an official veterinarian conducted a CFT test according to the Chilean control and eradication program instructions. Briefly, each animal was inoculated with $0.1 \mathrm{~mL}$ of commercial bovine tuberculin (CZV, Porriño, Spain) intradermally to then read out the reaction 72 hours after inoculation. Any increase in volume in the area was considered a positive result.

Gamma interferon test. The BOVIGAM ® commercial kit was used according to the manufacturers recommendations (Prionics AG).Two cut-off points were selected to define two interpretation criteria for the test as described by Alvarez et al (2012) ; the "severe interpretation" identified an animal as infected if the mean optical density (OD) of a sample stimulated with bovine PPD minus the mean OD of nil antigen was greater than 0.05 and greater than the same value of the sample stimulated with avian PPD; whereas the "standard interpretation" considered an animal as infected if the same value was above 0.1 and greater than the value obtained after stimulation with avian PPD. Avian and Bovine tuberculins used in this study were purchased from the same manufacturer. Additionally, in order to determine the cellular viability, Concanavalin-A was used as a cellular viability control, under the same processing protocol determined by the manufacturer for the standard processing.
M. bovis DNA extraction and purification method. DNA was extracted from lesions found on retropharyngeal and/ or mediastinal lymph nodes of animals presumptively infected with $M$. bovis by the cethyltrimethylammonium bromide (CTAB) method described by van Soolingen et al (1999), if no lesions were found on this target tissue, a pool of lymph node tissue was proccesed instead.

Real-Time PCR protocol for M. bovis confirmation. Real time PCR to identify $M$. bovis species was performed using a commercial kit (BOVIMAN $\left.{ }^{\circledR}\right)$, according to the manufacturer's instructions. Briefly, the PCR reaction included Master mix $10 \mu \mathrm{l}$, M. bovis P\&P $1 \mu \mathrm{l}$, PCR water $4 \mu \mathrm{l}$ and $5 \mu \mathrm{l}$ template DNA up to a total volume of $20 \mu \mathrm{l}$. The reactions were carried out in a Roche LightCycler 2.0 system under the following standard conditions: 1 cycle of $8 \mathrm{~min}$ at $95^{\circ} \mathrm{C}$ to continue with 45 denaturation cycles for $5 \mathrm{sec}$ at $95^{\circ} \mathrm{C}$, annealing for $12 \mathrm{sec}$ at $68^{\circ} \mathrm{C}$, and extension for $1 \mathrm{sec}$ at $72^{\circ} \mathrm{C}$. Negative and positive PCR controls were included.

\section{STATISTICAL ANALYSIS}

Two datasets were built based on how bTB infection status was defined. The first one (dataset for in vivo M. bovis diagnosis, $n=609$ ) included all animals where their bTB status was diagnosed using CFT and Gamma Interferon tests. The second (dataset for post mortem M. bovis diagnosis, $n=226$ ) included those animals in which the bTB status was confirmed by direct PCR on tissue samples.

The McNemar test was used to compare paired population proportions of positive results for the different diagnostic tools. The case definition for M. bovis-infected cattle was PCR confirmation of $M$. bovis from an individual tissue sample. The case definition for a non-infected individual was a cow originating from a herd where none of the animals tested were tissue PCR-positive. Apparent sensitivity was defined as the percentage of $M$. bovis-infected cattle testing CFT or Interferon positive. Apparent specificity was defined as the percentage of non- $M$. bovis-infected cattle testing CFT or Bovigam-negative. Both parameters were calculated using $M$. bovis PCR results as gold standard $(n=226)$. All statistical analyses were done using INFOSTAT.

\section{RESULTS AND DISCUSSION}

The analysis of the in vivo tests reported that CFT results classified 268 animals (44\%) as tuberculin reactors, however, on the same population, Interferon test showed only 99 samples as positive $(16.3 \%)$ using the standard interpretation, and when severe interpretation was used, the number rose to 118 animals (19.37\%) (table 1). The $\mathrm{P}$ value for the McNemar chi-square test showed that proportions of positive results for CFT, Interferon and direct PCR on tissue samples were significantly different $(P<0.001)$ (table 2), therefore the level of agreement 
Table 1. Number of cattle and tests results for M. bovis infection on Chilean dairy herds by CFT, Interferon and direct PCR.

\begin{tabular}{cccccc}
\hline Herd & $\mathrm{N}^{\circ}$ CFT $(+) /$ total & $\begin{array}{c}\mathrm{N}^{\circ} \text { Interferon } \\
(+) / \text { total }(\text { std })\end{array}$ & $\begin{array}{c}\mathrm{N}^{\circ} \text { Interferon } \\
(+) / \text { total }(\mathrm{sev})\end{array}$ & $\begin{array}{c}\mathrm{N}^{\circ} \text { non tested } \\
\text { for PM PCR }\end{array}$ & $\mathrm{N}^{\mathrm{o}}$ PCR $(+) /$ total \\
\hline 1 & $16 / 58$ & $2 / 58$ & $2 / 58$ & 31 & $8 / 27$ \\
2 & $1 / 1$ & $0 / 1$ & $1 / 1$ & 1 & $\mathrm{~N} / \mathrm{T}$ \\
3 & $7 / 18$ & $1 / 18$ & $2 / 18$ & 7 & $2 / 11$ \\
4 & $1 / 8$ & $0 / 8$ & $1 / 8$ & 1 & $2 / 7$ \\
5 & $43 / 43$ & $36 / 43$ & $39 / 43$ & 5 & $23 / 38$ \\
6 & $6 / 6$ & $1 / 6$ & $1 / 6$ & 6 & $\mathrm{~N} / \mathrm{T}$ \\
7 & $0 / 51$ & $0 / 51$ & $0 / 51$ & 0 & $0 / 51$ \\
8 & $48 / 48$ & $0 / 48$ & $2 / 48$ & 4 & $31 / 44$ \\
9 & $8 / 8$ & $2 / 8$ & $2 / 8$ & 6 & $1 / 2$ \\
10 & $71 / 71$ & $4 / 71$ & $8 / 71$ & 71 & $\mathrm{~N} / \mathrm{T}$ \\
11 & $5 / 91$ & $1 / 91$ & $3 / 91$ & 83 & $\mathrm{~N} / \mathrm{T}$ \\
12 & $27 / 38$ & $33 / 38$ & $35 / 38$ & 38 & $3 / 10$ \\
13 & $28 / 40$ & $16 / 40$ & $18 / 40$ & 30 & $\mathrm{~N} / \mathrm{T}$ \\
14 & $1 / 43$ & $0 / 43$ & $0 / 43$ & 43 & $\mathrm{~N} / \mathrm{T}$ \\
15 & $5 / 5$ & $2 / 5$ & $3 / 5$ & 5 & $\mathrm{~N} / \mathrm{T}$ \\
16 & $1 / 42$ & $1 / 42$ & $1 / 42$ & 42 & $85(\mathrm{~N}=226)$ \\
17 & $0 / 38$ & $0 / 38$ & $0 / 38$ & 10 & 383 \\
Total & $268(\mathrm{~N}=609)$ & $99(\mathrm{~N}=609)$ & $118(\mathrm{~N}=609)$ & & 5 \\
\hline
\end{tabular}

NT: not tested.

Table 2. Cross classification of CFT, Interferon (both versions) results for test comparison.

\begin{tabular}{lccccc}
\hline \multirow{2}{*}{ CFT results } & \multicolumn{2}{c}{ Interferon (std) } & & \multicolumn{2}{c}{ Interferon (sev) } \\
\cline { 2 - 3 } \cline { 5 - 6 } & Positive & Negative & & Positive & Negative \\
\hline Positive & 89 & 179 & & 106 & 162 \\
Negative & 10 & 331 & & 12 & 329
\end{tabular}

McNemar Chi-square test $(P<0.001)$.

between the results for each test was not determined by calculating the Kappa value. Using M. bovis PCR results as gold standard on the group of animals with full post mortem confirmation (226 animals), the apparent sensitivity of the CFT was 76.5\% (95\% CI: 66-85) instead of the apparent sensitivity for Interferon, which was $24.7 \%$ (95\% CI: 1635.3 ) and $29.4 \%$ (95\% CI: 20-40.3) for standard and severe interpretation, respectively. CFT apparent specificity was $70.9 \%$ (95\% CI: 62.7-78.3) and for Interferon was 90.07\%
(95\% CI 83.9-94.5) and 89.36\% (95\% CI: 83.1-93.9) for standard and severe interpretation, respectively (table 3 ).

Overall, intradermal tuberculin based diagnostics showed better performance when compared to Interferon, even though Interferon was more specific. Interferon based tests were presented as an alternative for bTB diagnosis (Bezos et al 2014b), and when compared with tuberculin based tests, showed positive characteristics that were supposed to be major improvements for the detection of infected animals. Among the advantages of the interferon based techniques over the intradermal tuberculin tests are less frequent handling of animals, objective numerical test results, and comparative response to two different antigens (Bezos et al 2014 , Lopes et al 2012, Faye et al 2011, Antognoli et al 2011) among others.

The major difference between previous surveys and the present report in relation to the supposed benefits of the use on Gamma interferon is that most of the above mentioned surveys were conducted under controlled experimental conditions and or in real field situations but with

Table 3. Cross classification of CFT and Interferon (both versions) and Direct PCR results (N=226), for apparent sensitivity and specificity estimation.

\begin{tabular}{|c|c|c|c|c|c|c|}
\hline \multirow{2}{*}{ PCR results } & \multicolumn{2}{|c|}{ CFT } & \multicolumn{2}{|c|}{ Interferon (std) } & \multicolumn{2}{|c|}{ Interferon (sev) } \\
\hline & Positive & Negative & Positive & Negative & Positive & Negative \\
\hline Positive & 65 & 20 & 21 & 64 & 25 & 60 \\
\hline Negative & 41 & 100 & 14 & 127 & 15 & 126 \\
\hline
\end{tabular}


high prevalence, which highlights a major difference from what was done in the present study. An inconsistent pattern for results obtained in this study was observed, with a big number of animals showing enormous variations of results, which could be explained due to risk factors such as animal age and herd geographic location (Gormley et al 2013). Furthermore, Gamma interferon depends on the presence of viable lymphocytes in the blood sample collected in the field; careful handling of blood samples and timely transportation to the laboratories are critical to this test and are limitations of this diagnostic tool (Antognoli et al 2011), for this reason, celular viability must be determined whenever this methodoloy is used to asses infection satus on herds, in the present study Concanavalin-A was used to assess this issue.

Due to the above mentioned limitations, specific cutoff lines for interferon based techniques for certain areas with particular low bTB prevalence should be considered (Faye et al 2011), as is the Chilean situation. In addition, the modification of the test with other more specific antigens (Faye et al 2011) and the use of better combinations of PPDs if in vitro testing is intended (Schiller et al 2010) must be taken into consideration.

The findings reported in this study raise the question: is it possible that in the previously described scenario of low bTB prevalence, high CFT false positive results and perhaps the presence of an unknown interfering mycobacterial infection, may be responsible for the poor performance of interferon based techniques in southern Chile?. The uncertain role of interference bacteria that could be affecting the results of the in vitro analysis must be considered, and perhaps this could explain the apparent lack of consensus of the results obtained in Chile versus the ones obtained elsewhere.

This simple and logical approach is widely demonstrated as mentioned earlier in several countries; however the role of other bacteria that could perhaps sensitize T-lymphocytes to respond to the avian PPD more vigorously than to the stimulation induced by bovine PPD, is not fully understood, at least for the Chilean situation. It is hypothesized that interfering bacteria could induce higher responses to the avian PPD, or weaker responses to bovine PPD, both situations ending on a misclassification of the tested animal.

Further studies are needed in order to assess this issue. It is well known that a big number of environmental mycobacteria species that inhabit soil infect domestic or wild ruminants such as the infection by Mycobacterium avium subsp. paratuberculosis, which is highly prevalent in cattle in southern Chile (Kruze et al 2013).

\section{ACKNOWLEDGEMENTS}

This work was supported by FIC De Los Ríos grant (2011). Eduardo Raffo was the recipient of a Doctoral Studentship from the Chilean government (CONICYT) during the present study and it was part of a Doctoral Thesis.

\section{REFERENCES}

Álvarez J, Pérez A,Bezos J, Marqués S, Grau A, et al. 2012. Evaluation of the sensitivity and specificity of bovine tuberculosis diagnostic tests in naturally infected cattle herds using a Bayesian approach. Vet Microbiol 155, 38-43.

Antognoli MC, Remmenga MD, Bengtson SD, Clark HJ, Orloski KA, et al. 2011. Analysis of the diagnostic accuracy of the gamma interferon assay for detection of bovine tuberculosis in U.S. herds. Prev Vet Med 101, 35-41.

Bezos J, Álvarez J, Romero B, de Juan L, Dominguez L. 2014ª Bovine tuberculosis: historical perspective. Res Vet Sci 97, S3-4.

Bezos J, Casal C, Romero B, Schroeder B, Hardegger R, et al. 2014 ${ }^{\mathrm{b}}$. Current ante-mortem techniques for diagnosis of bovine tuberculosis. Res Vet Sci 97, 44-52.

de la Rua-Domenech R, Goodchild AT, Vordermeier HM, Hewinson $\mathrm{RG}$, Christiansen KH, et al. 2006. Ante mortem diagnosis of tuberculosis in cattle: A review of the tuberculin tests, gamma-interferon assay and other ancillary diagnostic techniques. Res Vet Sci 81, 190-210.

Faye S, Moyen JL, Gares H, Benet JJ, Garin-Bastuji B, et al. 2011. Determination of decisional cut-off values for the optimal diagnosis of bovine tuberculosis with a modified IFNgamma assay (Bovigam(R)) in a low prevalence area in France. Vet Microbiol 151, 60-67.

Gormley E, Doyle M, Duignan A, Good M, More SJ, et al. 2013. Identification of risk factors associated with disclosure of false positive bovine tuberculosis reactors using the gamma-interferon (IFN gamma) assay. Vet Res 44.

Kruze J, Monti G, Schulze F, Mella A, Leiva S. 2013. Herd-level prevalence of Map infection in dairy herds of southern Chile determined by culture of environmental fecal samples and bulk-tank milk qPCR. Prev Vet Med 111, 319-324.

Lopes LB, Alves TM, Stynen APR, Mota PMPC, Leite RC, et al. 2012. Parameter estimation and use of gamma interferon assay for the diagnosis of bovine tuberculosis in Brazil. Pesqui Vet Bras 32, 279-283.

Max V, Paredes L, Rivera A, Ternicier C. 2011. National control and eradication program of bovine tuberculosis in Chile. Vet Microbiol 151, 188-191.

Neill SD, Skuce RA, Pollock JM. 2005. Tuberculosis-new light from an old window. J Appl Microbiol 98, 1261-1269.

Schiller I, Vordermeier HM, Waters WR, Kyburz A, Cagiola M, et al. 2010. Comparison of tuberculin activity using the interferon-gamma assay for the diagnosis of bovine tuberculosis. Vet Rec 167, 322-326.

Schiller I, Waters WR, Vordermeier HM, Jemmi T, Welsh M, et al. 2011. Bovine tuberculosis in Europe from the perspective of an officially tuberculosis free country: trade, surveillance and diagnostics. Vet Microbiol 151, 153-159.

van Soolingen D, Borgdorff MW, de Haas PE, Sebek MM, Veen J, Dessens M, et al. 1999. Molecular epidemiology of tuberculosis in the Netherlands: a nationwide study from 1993 through 1997. J Infect Dis 180, 726-736. 